\title{
Spontaneous rupture of the ureter
}

Alper $\underline{E k e n}^{1}, \mathrm{MD}, \mathrm{FEBU}$, Tugana $\underline{\text { Akbas }}^{2}, \mathrm{MD}$, Taner $\underline{\text { Arpaci }}^{2}, \mathrm{MD}$

ABSTRACT Spontaneous rupture of the ureter is a very rare condition and usually results from ureteral obstruction by a calculus. Only theoretical mechanisms have been proposed and no possible explanation has yet been reported in the literature. Intravenous contrast-enhanced computed tomography is the most informative study with high sensitivity. Treatment should be individualised, and depends on the state of the patient. Minimally invasive endourological procedures with double-J catheter placement and percutaneous drainage offer excellent results. Conservative management with analgesics and antibiotic coverage may be an alternative to surgery. Herein, we present a case of spontaneous rupture of the proximal ureter with no evidence of an underlying pathological condition.

Keywords: lithiasis, rupture, spontaneous, ureter, urinary extravasation

\section{INTRODUCTION}

Spontaneous rupture of the ureter is a very rare condition. Previously reported cases reflect an underlying pathology such as trauma, urinary calculi, obstruction, malignancy, idiopathic retroperitoneal fibrosis, or previous urinary tract surgery. ${ }^{1,2}$ Herein, we report a case of spontaneous rupture of the proximal ureter with no evidence of an underlying pathological condition.

\section{CASE REPORT}

A 29-year-old man presented to the emergency clinic with severe and progressive left flank pain accompanied by nausea and chills for the past six hours. Anamnesis revealed that he had no previous clinical history of any disease, including urinary complaints. On physical examination, he was oriented and cooperative, but distressed. He had left costovertebral angle tenderness. Bowel sounds were normal and there was no rebound or tenderness. His vital signs were normal: blood pressure 120/80 mmHg; cardiac rate 70 beats per min; and body temperature $36.7^{\circ} \mathrm{C}$. Laboratory evaluation revealed normal creatinine and blood urea nitrogen levels. Other biochemical results, including complete blood count, hepatic function tests, and C-reactive protein level, were also within normal ranges. Urine analysis showed 1-2 leucocytes and no erythrocytes per field.

Plain abdominal radiography was unremarkable, while abdominal ultrasonography showed mild left hydronephrosis. Initial, noncontrast-enhanced, multislice computed tomography (CT), performed with a 1-mm section thickness, demonstrated mild left hydronephrosis. No urinary calculus was seen (Fig. 1). Subsequently, contrast-enhanced CT showed a delay in left renal function, mild left hydronephrosis, and deformation of the ureteropelvic junction. Routinely, we obtained delayed scans through the kidneys at 7-min intervals. To opacify the left collecting system, we had to repeat delayed scans through the ureters at 15-min intervals. With the coronal and sagittal reformats of the delayed phase, extravasation of contrast medium around the left upper ureter and ureteropelvic junction was demonstrated clearly, with no visible evidence of any obstruction or tumour

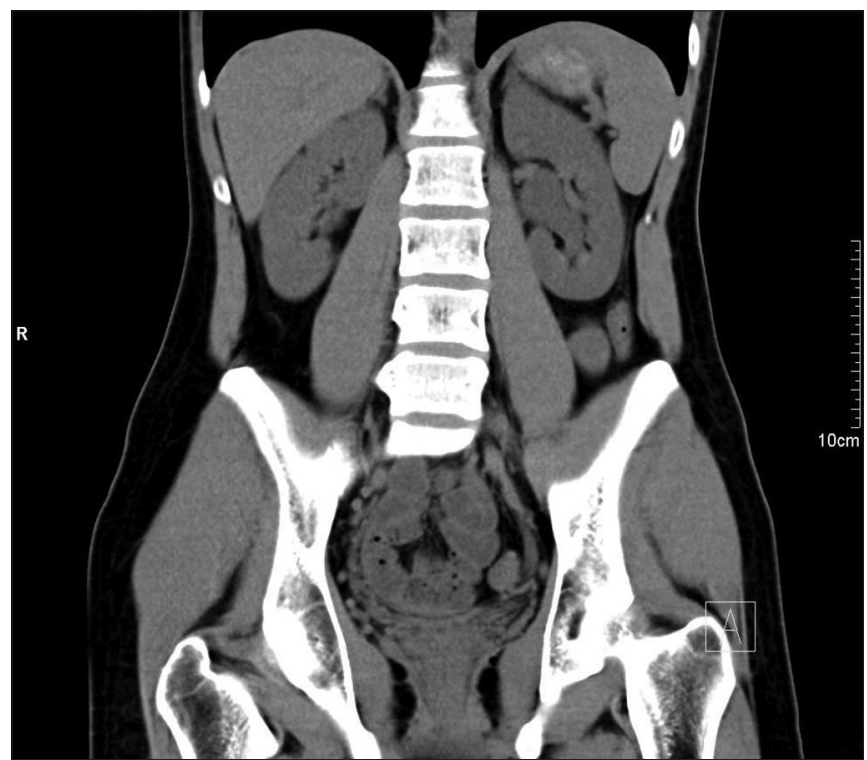

Fig. 1 Precontrast coronal CT image shows mild left hydronephrosis without urinary calculi.

(Figs. 2 \& 3). Based on CT and perioperative pyelography, the patient was diagnosed with grade 2 partial ureter rupture, according to the ureteral injury scale proposed by Moore et al..$^{(3)}$

The patient was initially managed by an endourological procedure and a double-J catheter was inserted. His Foley catheter was removed the next day and he was discharged with no complications. The double-J catheter was removed in the second postoperative month and retrograde pyelography revealed no extravasation. Follow-up contrast-enhanced CT performed in the sixth postoperative month was normal.

\section{DISCUSSION}

Spontaneous rupture of the ureter is a rare condition and no plausible explanation has yet been published in the literature; only theoretical mechanisms have been proposed. Impaction of stones on the ureter wall, or a downward moving calculus, which may cause erosion and ulceration of the ureteral wall, may lead to ureteral rupture at the distal ureteral obstruction..$^{(2)}$ In addition,

${ }^{1}$ Urology Department, ${ }^{2}$ Department of Radiology, Acıbadem Hastanesi, Adana, Turkey

Correspondence: A/Prof Alper Eken, Consultant, Urology Department, Acıbadem Hastanesi, Cumhuriyet Caddesi, 01130 Adana, Turkey. alpereken@yahoo.com 


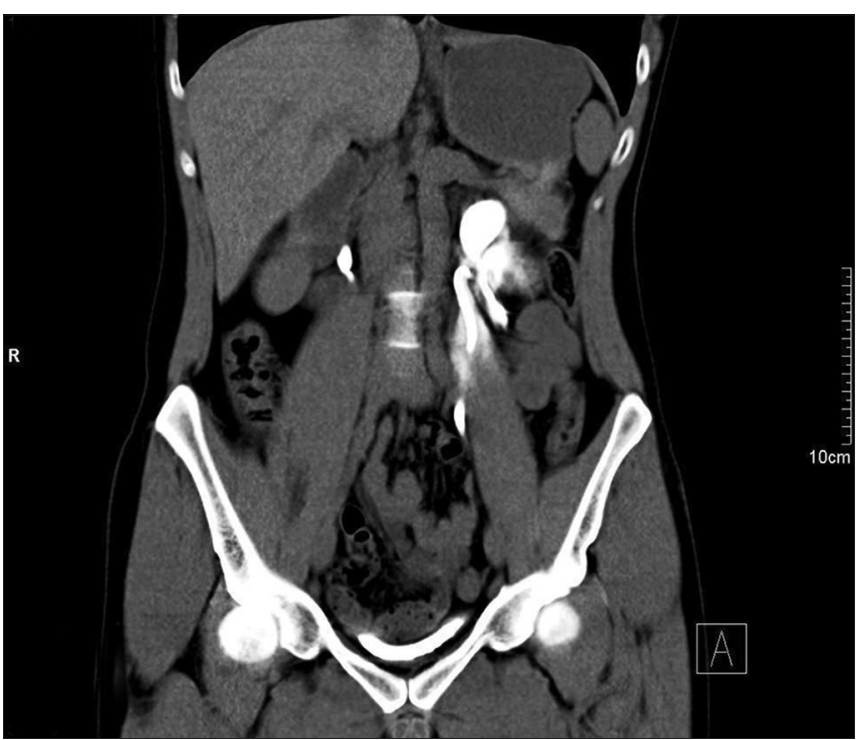

Fig. 2 Postcontrast coronal CT image shows contrast extravasation from the left upper ureter.

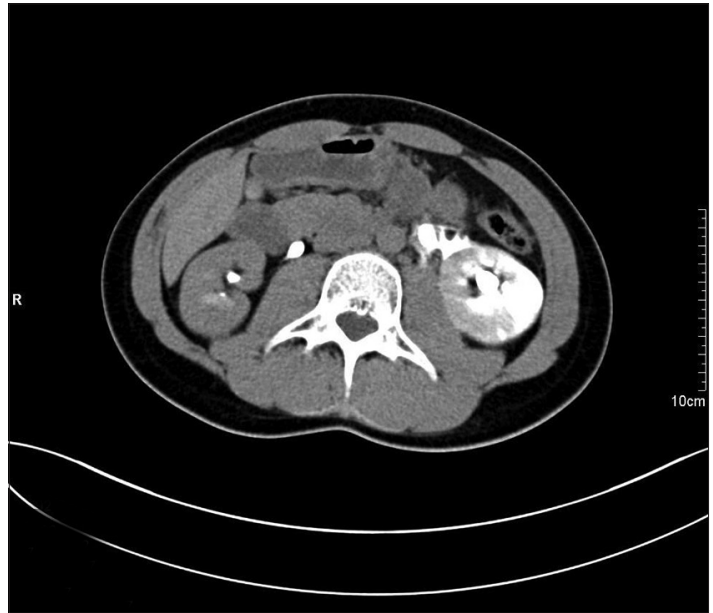

Fig. 3 Postcontrast axial CT image shows leakage of radiocontrast media at the level of the upper ureter.

malignancy, ${ }^{(4)}$ idiopathic retroperitoneal fibrosis, ${ }^{(5)}$ posterior urethral valves or bladder outlet obstruction, ${ }^{(6)}$ and some connective tissue disorders causing fibrotic changes such as Klinefelter syndrome ${ }^{(7)}$ have also been proposed to be causes of spontaneous rupture of the ureter. Spontaneous rupture of the ureter during kidney biopsy, ${ }^{(8}$ as a complication of extracorporeal shock wave lithotripsy, ${ }^{\left({ }^{9}\right)}$ and increased urine flow from a fluid bolus ${ }^{(10)}$ have also been reported. In the present case, no evidence of any underlying pathological condition was shown. Although asked several times, the patient denied having any previous calculi or trauma history, or recognising that he had passed out any calculi. During cystoscopic evaluation, no evidence of lower urinary tract obstruction was observed. No plausible explanation for the cause of our patient's ureteric rupture was found. However, the most feasible theory was that a small calculus that had caused transient acute obstruction was subsequently passed out spontaneously without the patient realising it.

Schwartz et al reported a case of rupture of the ureter and used the term 'spontaneous' when there was: (a) no external trauma; (b) no cystoscopic ureteral manipulation; (c) no external compression; (d) an absence of destructive kidney disease; and (e) no history of any previous surgery. ${ }^{(11)}$ Koga et al reported 11 cases of spontaneous peripelvic extravasation. ${ }^{(12)}$ However, nine of the patients had urinary obstruction with calculi, one had invasion by a sigmoid carcinoma, and one had ureteral transitional cell carcinoma. Stravodimos et al reported five cases of spontaneous rupture; one patient had a stone, but four had no obvious cause of rupture. ${ }^{(13)}$ Choi et al reported a case of upper ureteral rupture, caused by urinary retention due to neurogenic bladder, ${ }^{(14)}$ and Yoshii et al reported a patient with malignant lymphoma, which was the cause of spontaneous ureteral rupture. ${ }^{(15)}$

All of the aforementioned reports show that 'spontaneous' ureteric rupture typically occurs secondary to a ureteric stone, inside or outside the ureter. Migration of the stone fragments outside of the ureter or retroperitoneal space is a complication of ureteroscopy or percutaneous nephrolithotomy, with a $0.2 \%$ incidence rate. Removal of these paraureteral stones is not required and the condition can be managed conservatively. However, paraureteral stones can rarely lead to the formation of ureteral strictures or granulomas that may cause recurrent ureteral obstruction or spontaneous rupture of the ureter. ${ }^{(16)}$ Our patient had no previous stone surgery, and no ureteral or paraureteral calculi was demonstrated on noncontrast-enhanced CT.

Spontaneous rupture of the ureter has no characteristic clinical signs. On physical examination, patients may have abdominal tenderness and pain, with costovertebral angle tenderness on the ipsilateral side. In some cases, the diagnosis can be difficult because of the nonspecific symptoms. The differential diagnosis includes urinary lithiasis, appendicitis, cholecystitis, diverticulitis, and other possible causes of abdominal pain. Ureteral rupture often presents with gastrointestinal symptoms in close proximity to the peritoneum and can cause chemical peritoneal irritation, mimicking diverticulitis or appendicitis. ${ }^{(3)}$ Sudden obstruction of the ureter increases the pressure of the collecting system and causes renal colic. In the present case, if pain were related to pressure elevation in the collecting system, we would expect a decrease in pain after ureteral rupture. However, no decrease in pain had occurred. This could be due to urine extravastion in the perianal fat causing an inflammatory response, which triggers pain through the activation of primary sensory neurons, as well as the subsequent release of inflammatory neuropeptides. Nevertheless, we could not identify with certainty the cause and aetiology of the flank pain in our patient.

Intravenous pyelography is a useful tool for visualising the urinary tract. Contrast media extravasation and ultrasonography can also be used to search for other abdominal anomalies. However, intravenous contrast-enhanced $\mathrm{CT}$ is the most informative modality and has a higher sensitivity than other imaging modalities. The location and degree of leakage can also be readily assessed by $\mathrm{CT}$. False negative results are reduced based on the results of abdominal plain radiography in the excretory phase. In our patient, we diagnosed ureteral rupture via the extravasation of contrast medium from the perforated ureter with delayed contrast-enhanced CT. 
Combined with retrograde pyelography, thin-section delayed CT urography is the first-line imaging technique with the highest diagnostic accuracy for upper urinary tract urothelial cell carcinoma. Direct visual inspection via rigid or flexible ureteroscopy is useful when there is diagnostic uncertainty of ureteral transitional cell carcinoma (TCC). Microscopic haematuria is the most common symptom of upper tract TCC, but our patient's urinary analysis did not reveal any haematuria. In the present case, ureteroscopy was not planned at admission because the procedure may increase the degree of rupture and can cause iatrogenic avulsion, which is more dramatic than partial rupture. After retrograde pyelography and previous CT urography were considered together, we did not perform ureteroscopy during the removal of the double-J catheter. If there is any suspicion of upper urinary tract cancer, ureteroscopy (especially the flexible type) needs to be carried out. Cytology can assist in the diagnosis of upper urinary tract cancer, but the sensitivity of urinary cytology correlates closely with the pathologic tumour grade. For low-grade papillary tumours of the upper urinary tract, urinary cytology yields very low sensitivity and specificity, as low as $10 \%$ and $50 \%$, respectively. ${ }^{(17)}$ In spite of its low sensitivity and specificity, urinary cytology should be undertaken to rule out upper tract cancer, even in the case of our patient.

Spontaneous ureteral perforation can have serious consequences, including urinoma, perinephric or retroperitoneal abscess formation, and urosepsis. Consequently, spontaneous ureter rupture should be treated promptly. Treatment should be individualised, and depends on the state of the patient and the ureteral rupture grade. Antibiotic coverage is mandatory for all patients. In the present case, a double-J catheter was placed under fluoroscopic control. The symptoms regressed within a few hours and the patient was discharged after 24 hours. Stravodimos et al, who reported the successful treatment of ureteric rupture by insertion of a double-J stent under fluoroscopy, likewise achieved unobstructed urinary outflow, healing of the perforation, and stabilisation and gradual absorption of the urinoma. ${ }^{(13)}$ Although there have been reports of ureteral rupture being managed via open surgery, many studies have also highlighted the significant benefits of minimally invasive endourological procedures such as double-J catheter placement and percutaneous drainage. ${ }^{(6-8,18)}$ Recently, successful conservative management with analgesics and antibiotic coverage has been reported in the literature. These studies recommended nonoperative treatments in stable patients, but advised that endourological intervention may still be necessary if conservative management fails..$^{(3,4,18)}$
In conclusion, spontaneous rupture of the ureter is a rare entity that can present with no evidence of any underlying pathological condition. Ureteral perforation can be detected using delayed contrast-enhanced imaging techniques. As rupture of the ureter can cause serious complications, including urinoma, retroperitoneal abscess formation and sepsis, prompt treatment of the condition is therefore necessary.

\section{REFERENCES}

1. Ay D, Yencilek E, Celikmen MF, Akkas M, Ekci B. Spontaneous rupture of ureter: an unusual cause of acute abdominal pain. Am J Emerg Med 2012; 30:390.e1-2.

2. Liu S, Lin J, Huang C, Tsai I. Spontaneous rupture of the ureter mimicking acute appendicitis: Two case reports. JACME 2011; 1:61-3.

3. Moore EE, Cogbill TH, Jurkovich GJ, et al. Organ injury scaling. III: Chest wall, abdominal vascular, ureter, bladder, and urethra. J Trauma 1992; 33:337-9.

4. Inahara M, Kojima S, Takei K, et al. [Two cases of spontaneous rupture of upper urinary tract caused by the primary ureteral or renal pelvic tumor: a case report]. Hinyokika Kiyo 2009; 55:31-4. Japanese.

5. Caruso Lombardi A, Rinaldi MF, Bartalena T, Elmi F, Mughetti M. Urinary ascites due to retroperitoneal fibrosis: a case report. Acta Radiol 2007; 48:119-21.

6. Akpinar H, Kural AR, Tüfek I, et al. Spontaneous ureteral rupture: is immediate surgical intervention always necessary? Presentation of four cases and review of the literature. J Endourol 2002; 16:179-83.

7. Reva S, Tolkach Y. Spontaneous pelvic rupture as a result of renal colic in a patient with klinefelter syndrome. Case Rep Urol 2013; 2013:374973.

8. Chen SC, Liu WC, Wang CS, et al. Iatrogenic rupture of the ureter during kidney biopsy. Kaohsiung J Med Sci 2010; 26:502-5.

9. Trottmann $M$, Tritschler S, Graser A, et al. [Injuries of the renal pelvis and ureter. Diagnosis and management]. Urologe A 2007; 46:927-34. German.

10. Huang E, Sayegh R, Craigo S, Chelmow D. Rupture of the renal pelvis associated with intravenous fluid bolus. J Matern Fetal Neonatal Med 2002; 11:345-6.

11. Schwartz A, Caine M, Hermann G, Bittermann W. Spontaneous renal extravasation during intravenous urography. Am J Roentgenol Radium Ther Nucl Med 1966; 98:27-40.

12. Koga S, Arakaki Y, Matsuoka M, Ohyama C. Spontaneous peripelvic extravasation of urine. Int Urol Nephrol 1992; 24:465-9.

13. Stravodimos K, Adamakis I, Koutalellis G, et al. Spontaneous perforation of the ureter: clinical presentation and endourological management. J Endourol 2008; 22:479-84.

14. Choi SK, Lee S, Kim S, et al. A rare case of upper ureter rupture: ureteral perforation caused by urinary retention. Korean J Urol 2012; 53:131-3.

15. Yoshii T, Horiguchi A, Shirotake S, et al. [Spontaneous rupture of the ureter as the primary symptom of malignant lymphoma]. Hinyokika Kiyo 2010; 56:639-43. Japanese.

16. Geavlete P, Georgescu D, Niță G, Mirciulescu V, Cauni V. Complications of 2735 retrograde semirigid ureteroscopy procedures: a single-center experience. J Endourol 2006; 20:179-85.

17. Brimo F, Vollmer RT, Case B, et al. Accuracy of urine cytology and the significance of an atypical category. Am J Clin Pathol 2009; 132:785-93.

18. Chen $\mathrm{CC}$, Chang $\mathrm{CH}$, Liu YL, Liu JH, Huang CC. A tiny stone induced ureteral rupture. Urolithiasis. Ann Acad Med Singapore 2010; 39:948-2. 